\title{
A Dynamic Spatial Model of Conflict Escalation
}

\author{
P. Baudains ${ }^{* \dagger} \quad$ H. M. Fry ${ }^{\ddagger} \quad$ T. P. Davies ${ }^{* \dagger} \quad$ A. G. Wilson ${ }^{\ddagger} \quad$ S.R. Bishop ${ }^{\dagger}$
}

August 10, 2015

\begin{abstract}
In both historical and modern conflicts, space plays a critical role in how interactions occur over time. Despite its importance, the spatial distribution of adversaries has often been neglected in mathematical models of conflict. In this paper, we propose an entropy-maximising spatial interaction method for disaggregating the impact of space, employing a general notion of 'threat' between two adversaries. This approach addresses a number of limitations that are associated with partial differential equation approaches to spatial disaggregation. We use this method to spatially disaggregate the Richardson model of conflict escalation, and then explore the resulting model with both analytical and numerical treatments. A bifurcation is identified that dramatically influences the resulting spatial distribution of conflict and is shown to persist under a range of model specifications. Implications of this finding for real-world conflicts are discussed.
\end{abstract}

${ }^{*}$ UCL Department of Security and Crime Science, University College London, 35 Tavistock Square, London, WC1H 9EZ, UK

${ }^{\dagger}$ UCL Department of Mathematics, University College London, Gower Street, London, WC1E 6BT, UK

${ }_{\ddagger}^{\ddagger}$ UCL Centre for Advanced Spatial Analysis, University College London, Gower Street, London, WC1E 6BT, UK 


\section{Introduction}

The spatial distribution of resources can have a significant effect on the actions of adversaries during conflict. Mathematical models that incorporate such factors can help in the consideration of strategies for deployment over space or the forecasting of those geographic regions that may be more at risk. Historically, dynamic ecological models of interacting populations have been used extensively to investigate conflict between adversaries, but have typically neglected the influence of space. Examples include the Richardson model of conflict escalation (Richardson, 1960), the Lanchester equations of attrition warfare (Lanchester, 1916), and the Lotka-Volterra model for predator-prey interactions, originally described as an ecological model in Lotka (1925). Since these landmark publications, a number of studies have sought to develop these models and insights have been obtained into different types of conflict (Deitchman, 1962; Jackson et al., 1978; Zinnes and Muncaster, 1984; Intriligator and Brito, 1988; Karmeshu et al., 1990; Saperstein, 2007; Liebovitch et al., 2008; Blank et al., 2008; Atkinson et al., 2011; Qubbaj and Muneepeerakul, 2012; Rojas-Pacheco et al., 2013; Kress and MacKay, 2014), but explicit modelling of spatial interactions is rarely considered. By neglecting space, it is implicitly assumed that total resource levels are the main drivers that govern the system, but it may be that, in fact, the distribution of resources has a material effect on real-life outcomes.

When models of conflict have incorporated some form of spatial dependence, it is often modelled by adding reaction-diffusion dynamics to a system of ordinary differential equations (Epstein, 1997; Brantingham et al., 2012). Some have argued, however, that reaction-diffusion models may not always be the most appropriate method of accounting for such dependence (Durrett and Levin, 1994; Ilachinski, 2004; González and Villena, 2011). Diffusive dynamics, in which conflict is modelled as spreading from areas of high concentration to low, may not always correspond to real-world spatial interactions.

Other studies have incorporated other mechanisms, such as advection, in an effort to construct more realistic models (Protopopescu and Santoro, 1989; Short et al., 2010a,b; Pitcher, 2010; Keane, 2011). Such models still rely on a number of restrictive assumptions. For instance, they require smooth dependent variables in space in order to ensure existence of second partial derivatives. Since data is often aggregated into discrete geographic areas, this requires the construction of kernel density estimators, which, in turn, 
requires further modelling assumptions regarding the choice of estimator and associated parameters.

Discontinuities in space are also likely to be encountered, for example via physical (rivers and roads) or geopolitical boundaries, which can contradict assumptions of smooth dependent variables (although some techniques have been developed to account for such effects (Smith et al., 2010)).

Finally, solutions to partial differential equations require the specification of spatial boundary conditions, which, in many cases, may be difficult to define if the spatial area of interest has no natural boundary that contains the dynamics.

To address these limitations, we propose an entropy maximising approach to the spatial disaggregation of conflict models. We demonstrate this approach by disaggregating the Richardson model of conflict escalation. In what follows, we first outline the Richardson model before presenting this disaggregation. We then consider the resulting model in a range of scenarios. Starting with highly simplified scenarios, for which the behaviour of the model can be analytically determined, the complexity of the model is increased by considering higherdimensional phase spaces and corresponding parameter spaces. This leads to an understanding of the model's behaviour and implications in more general scenarios.

\section{The Richardson model}

The Richardson model was initially conceived as a model of arms expenditure between two nations in the lead up to war (Richardson, 1960). As a consequence, the dependent variables, given here by $p$ and $q$, were taken to be the levels of military expenditure of two nations. The model is given by the following two-dimensional linear system of ordinary differential equations:

$$
\begin{aligned}
& \frac{d p}{d t}=\dot{p}=-\sigma_{1} p+\rho_{1} q+\epsilon_{1} \\
& \frac{d q}{d t}=\dot{q}=\rho_{2} p-\sigma_{2} q+\epsilon_{2},
\end{aligned}
$$

where parameters $\sigma_{1}$ and $\sigma_{2}$ determine the influence on the change in military expenditure proportional to existing expenditure, and $\rho_{1}$ and $\rho_{2}$ determine the rate of the action-reaction relationship between the two adversaries. The terms $\epsilon_{1}$ and $\epsilon_{2}$ are those associated with external grievances. Typically, $\rho_{1}$ and $\rho_{2}$ will 
be positive, as an increase in military defences of one side spurs increased defences of the other. $\sigma_{1}$ and $\sigma_{2}$ are also typically positive: Richardson hypothesised that there will be some inhibition associated with an increasing military arsenal, perhaps through pressures placed upon the government of each nation by their electorate. The inhibition terms may also be interpreted as the rate of depreciation of existing arms.

Richardson showed that if $\rho_{1} \rho_{2}<\sigma_{1} \sigma_{2}$ then there is a unique positive equilibrium in the plane, which is globally attractive. In this case, $p$ and $q$ are constrained more by their internal dynamics (with intensity given by the parameters $\sigma_{1}$ and $\sigma_{2}$ ) than by the interaction terms (with intensity given by $\rho_{1}$ and $\rho_{2}$ ). If this condition does not hold, then the equilibrium is a saddle, and escalatory dynamics such as arms races can occur.

It has been argued elsewhere that the model represents a very general conflict escalation process (Zinnes and Muncaster, 1984) and, as such, can be considered to model a wide range of potential systems in which two adversaries are subject to retaliation (see also Liebovitch et al. (2008), who present a nonlinear variant of Richardson's model to consider general conflict processes). Consequently, for the remainder of this article, the dependent variables $p$ and $q$ are taken to be a more general and abstract measure of hostility between two adversaries.

Although Richardson's model was not spatially explicit, he was highly interested in spatial and geographic problems such as the measurement of borders and population density (Richardson (1961); see also Mandelbrot (1967)). He also considered how such measures might influence conflict in his work on deadly quarrels (Richardson, 1952).

\section{A spatially-explicit Richardson model}

Spatial interaction models have been employed previously within both static and dynamic spatial models to consider retail systems (Harris and Wilson, 1978; Wilson, 2008); international migration (Dennett and Wilson, 2013); rioting (Davies et al., 2013); international trade (Fry and Wilson, 2012) and ecological dynamics (Wilson, 2006). They have been empirically shown to provide plausible accounts of spatial processes 
for many of these examples.

To begin, we consider a two-dimensional manifold $\mathcal{M}$, on which conflict between two adversaries takes place. Suppose that one adversary is located at the points $\mathbf{x}_{1}, \mathbf{x}_{2}, \ldots, \mathbf{x}_{N} \in \mathcal{M}$, while their opponent is located at $\mathbf{y}_{1}, \mathbf{y}_{2}, \ldots, \mathbf{y}_{M} \in \mathcal{M}$. In other words, each adversary is discretely distributed over $\mathcal{M}$, perhaps due to the positions of military bases, allied settlements, or gang safe houses, depending on the application of the model.

In order to maintain generality, the dependent variables for the system are taken to be general measures of conflict, hostility or defence capability of each adversary. In the disaggregated system, these measures are tracked individually at each location. In other words, the variables to be considered are $p_{1}, p_{2}, \ldots, p_{N}$, which correspond to levels of hostility associated with locations $\mathbf{x}_{1}, \mathbf{x}_{2}, \ldots, \mathbf{x}_{N}$, respectively, and $q_{1}, q_{2}, \ldots, q_{N}$, which correspond to levels of hostility associated with locations $\mathbf{y}_{1}, \mathbf{y}_{2}, \ldots, \mathbf{y}_{M}$, respectively.

It is assumed similar mechanisms to the original Richardson model influence the variable $p_{j}$, for each index $j$. That is, $\dot{p}_{j}$ depends on three terms as in equation (1): the action-reaction term that itself depends on the adversary who is distributed over the manifold, representing the retaliatory dynamics driving the system; a measure of inhibition, representing each adversary's natural inclination to avoid conflict; and external grievances that may be present at $\mathbf{x}_{j}$.

The action-reaction term within the equation for $\dot{p}_{j}$ is assumed to depend on the variables $q_{1}, q_{2}, \ldots, q_{M}$, representing the level of hostility of their adversary. In particular, it is proposed that this term is given by a weighted sum of these terms, with corresponding weighting factors $w_{l j} \in[0,1]$, which serve to specify the proportion of $q_{l}$ that contributes to the action-reaction dynamics of $p_{j}$ for every $l$ and $j$. These weighting factors will be modelled explicitly in what follows. Following Richardson, the second term, representing inhibition mechanisms, is taken to be proportional to the hostility $p_{j}$ and the third term, representing external grievances associated with the hostility $p_{j}$, is taken to be a constant.

With an analogous equation for $\dot{q}_{l}$, for some index $l$, but with corresponding action-reaction weighting 
factors denoted by $v_{j l}$, the spatial Richardson model is

$$
\begin{aligned}
& \dot{p}_{j}=-\sigma_{1} p_{j}+\rho_{1} \sum_{l=1}^{M} q_{l} w_{l j}+\epsilon_{1} \iota_{j}, \quad j=1, \ldots, N, \\
& \dot{q}_{l}=\rho_{2} \sum_{j=1}^{N} p_{j} v_{j l}-\sigma_{2} q_{l}+\epsilon_{2} \kappa_{l}, \quad l=1, \ldots, M,
\end{aligned}
$$

where, as before, $\rho_{1}$ and $\rho_{2}$ specify the intensity of the action-reaction dynamics, $\sigma_{1}$ and $\sigma_{2}$ specify the extent to which there is inhibition to growth in hostility, and $\epsilon_{1} \iota_{j}$ and $\epsilon_{2} \kappa_{l}$ are the levels of external grievance associated with $p_{j}$ and $q_{l}$, respectively.

Since the model is a disaggregation of the full Richardson model, it is assumed that the dynamics of the aggregated system - that is, the system defined by the hostility of each adversary as a whole - follow the original Richardson dynamics. Thus, it is assumed that

$$
\sum_{j=1}^{N} w_{l j}=1, \quad l=1, \ldots, M ; \quad \sum_{l=1}^{M} v_{j l}=1, \quad j=1, \ldots, N,
$$

and that

$$
\sum_{j=1}^{N} \iota_{j}=1, \quad \sum_{l=1}^{M} \kappa_{l}=1
$$

Setting $p=\sum_{j=1}^{N} p_{j}$ and $q=\sum_{l=1}^{M} q_{l}$, it can be seen that the aggregation of the system in equation (2), obtained by summing the derivatives of each component, is equivalent to the system in equation (1).

In order to derive an explicit form for the model, further assumptions are required. It is assumed that $\iota_{j}=1 / N$ and $\kappa_{l}=1 / M$, so that external grievances impact $p_{j}$ and $q_{l}$ similarly over different values of $j$ and l. This assumption can be easily generalised, although such generalisations are not considered in the present article.

Equation (3) and the requirement that the weightings $w_{l j} \in[0,1]$ imply that the problem of finding an explicit analytical expression for $w_{l j}$ is equivalent to finding a probability distribution describing the probability that $\mathbf{x}_{j}$ is the location towards which a resource at $\mathbf{y}_{l}$ (with hostility $q_{l}$ ) will exert its threat. To do this, we employ the principal of maximum entropy (Wilson, 1970; Senior, 1979) to find the probability distribution that is least biased with respect to some modelling assumptions, or constraints, which we state in what follows. 
To specify these constraints, we define a metric $d: \mathcal{M} \times \mathcal{M} \rightarrow \mathbb{R}$ such that, for any two locations $\mathbf{x}_{j}, \mathbf{y}_{l} \in \mathcal{M}, d\left(\mathbf{x}_{j}, \mathbf{y}_{l}\right)$ is a measure of impedance, distance, or travel cost between $\mathbf{x}_{j}$ and $\mathbf{y}_{l}$. We fix the average cost of exerting threat in the system, so that:

$$
\sum_{l=1}^{M} \sum_{j=1}^{N} w_{l j} d\left(\mathbf{x}_{j}, \mathbf{y}_{l}\right)=c_{1},
$$

for some positive constant $c_{1}$. This constraint formulates Tobler's first law of geography in the model, forcing nearer things to be more related than farther things (Tobler, 1970) and, therefore, more threat to be exerted between opponents that are nearby.

Additionally, we assume that there is some incentive $b_{j}$ in addition to the cost of transporting threat to $\mathbf{x}_{j}$ from $\mathbf{y}_{l}$ (as given by $\left.d\left(\mathbf{x}_{j}, \mathbf{y}_{l}\right)\right)$ that can be offset against $d\left(\mathbf{x}_{j}, \mathbf{y}_{l}\right)$. It is assumed that targets with a higher threat incentive can have threat exerted on them even when the cost of exerting this threat is high. Similarly to equation (5), we fix the average cost of exerting threat in the system, offsetting the added benefit that might be obtained by the incentive measure, as

$$
\sum_{l=1}^{M} \sum_{j=1}^{N} w_{l j}\left(d\left(\mathbf{x}_{j}, \mathbf{y}_{l}\right)-b_{j}\right)=c_{2},
$$

for some constant $c_{2}$.

Finally, we assume that the incentive to exert threat on $\mathbf{x}_{j}$ is proportional to the logarithm of the hostility $p_{j}$. Thus, locations $\mathbf{x}_{j}$ with higher levels of hostility will provide more incentive for threats to travel longer distances as these locations will represent more of a threat to opponents at $\mathbf{y}_{l}$. A logarithm function is used so that this added incentive has diminishing returns to scale. Using equation (5) and equation (6), we obtain:

$$
\sum_{l=1}^{M} \sum_{j=1}^{N} w_{l j} \ln p_{j}=c_{3},
$$

for some constant $c_{3}$. This constraint is often employed in similar production-constrained spatial interaction models to reflect the added benefit associated with interacting with a particular target (Wilson, 1967, 2008; Davies et al., 2013).

Following Wilson (1970), the values for the weightings $w_{l j}$ for $l=1, \ldots, M$ and $j=1, \ldots, N$ can be determined by analogy with a problem in information theory, where a system comprised of a large number 
of very small distinct units originating at locations $\mathbf{x}_{j}$ must be assigned to destinations $\mathbf{y}_{l}$. In previous applications of the model, the individual units that flow have included money and people; however, for the present purposes, in which a general model of conflict is sought, the quantity flowing from $i$ to $j$ is assumed to be a conceptual measure of 'threat'. This is a novel interpretation of the following well-known derivation of the model, and, as will be demonstrated, enables a link between this modelling framework and a range of conflict models, such as the spatial Richardson model to which it is applied here.

When framed in this light, it can be shown that the most likely outcome for $w_{l j}$ may be determined via maximisation of the function:

$$
S=-\sum_{l=1}^{M} \sum_{j=1}^{N} w_{l j} \ln w_{l j},
$$

where $S$ is the sum of the Shannon entropy for probability distributions $\left\{w_{l j}\right\}_{j=1,2, \ldots, N}$ for $l=1,2, \ldots, M$ (see Senior (1979) for more detail), subject to constraints in equations (3), (5) and (7). Using the method of Lagrangian multipliers, this obtains

$$
w_{l j}=\frac{p_{j}^{\alpha} e^{-\beta d\left(\mathbf{x}_{j}, \mathbf{y}_{l}\right)}}{\sum_{j^{\prime}=1}^{N} p_{j^{\prime}}^{\alpha} e^{-\beta d\left(\mathbf{x}_{j^{\prime}}, \mathbf{y}_{l}\right)}},
$$

for parameters $\alpha$ and $\beta$, and where the subscript $j^{\prime}$ has been introduced to distinguish it from $j$. The weighting $w_{l j}$ determines the extent to which $q_{l}$ influences the reactive retaliatory behaviour of $p_{j}$. It can be interpreted as a weighted comparison of $p_{j}$ against $p_{j^{\prime}}$ for $j^{\prime}=1,2, \ldots N$, with each weighted according to the distance to the adversary at $\mathbf{y}_{l}$.

By an analogous derivation, a similar expression may be derived for the retaliatory effect on $q_{l}$ from $p_{j}$, with corresponding weightings $v_{j l}$, given by

$$
v_{j l}=\frac{q_{l}^{\gamma} e^{-\delta d\left(\mathbf{y}_{l}, \mathbf{x}_{j}\right)}}{\sum_{l^{\prime}=1}^{M} q_{l^{\prime}}^{\gamma} e^{-\delta d\left(\mathbf{y}_{l^{\prime}}, \mathbf{x}_{j}\right)}},
$$

for further new parameters $\gamma$ and $\delta$, and subscript $l^{\prime}$.

Returning to equation (2), the spatially-explicit Richardson model for two adversaries discretely dis- 
tributed over a manifold $\mathcal{M}$ with associated distance metric $d: \mathcal{M} \times \mathcal{M} \rightarrow \mathbb{R}$ is, therefore, given by

$$
\begin{aligned}
& \dot{p}_{j}=-\sigma_{1} p_{j}+\rho_{1} \sum_{l=1}^{M} q_{l} \frac{p_{j}^{\alpha} e^{-\beta d\left(\mathbf{x}_{j}, \mathbf{y}_{l}\right)}}{\sum_{j^{\prime}}^{N} p_{j^{\prime}}^{\alpha} e^{-\beta d\left(\mathbf{x}_{j^{\prime}}, \mathbf{y}_{l}\right)}}+\frac{\epsilon_{1}}{N} \\
& \dot{q}_{l}=-\sigma_{2} q_{l}+\rho_{2} \sum_{j=1}^{N} p_{j} \frac{q_{l}^{\gamma} e^{-\delta d\left(\mathbf{y}_{l}, \mathbf{x}_{j}\right)}}{\sum_{l^{\prime}}^{M} q_{l^{\prime}}^{\gamma} e^{-\delta d\left(\mathbf{y}_{l^{\prime}}, \mathbf{x}_{j}\right)}}+\frac{\epsilon_{2}}{M}
\end{aligned}
$$

for $j=1,2, \ldots, N$ and $l=1,2, \ldots, M$.

Equation (11) extends the version of Richardson's model in equation (1) by explicitly incorporating the impact of space via the metric $d$. Advantages over other methods of modelling spatial conflict processes (such as partial differential equations or multi-agent simulations) include the explicit and relatively general assumptions required to derive the model (without a reliance on the continuity of the locations of adversaries), together with its concise analytical form, enabling the model to be interrogated analytically to obtain insights. Since few restrictions have been placed upon the distance metric, the model can be applied to a range of conflict processes involving retaliatory dynamics and spatial interaction.

\section{Analysis}

As a first step, some simplifying assumptions are made. In what follows, a reduced parameter space is considered in which $\alpha=\gamma=1, \delta=\beta$, and $\sigma_{1}=\sigma_{2}=\sigma$. These imply: that $\dot{p}$ and $\dot{q}$ depend linearly on $p$ and $q$ respectively in both the numerator and denominator of the action-reaction term (which combine to form a nonlinear function); that both adversaries react to impedance on $\mathcal{M}$ at the same rate; and that both adversaries react to internal constraints at the same rate. In accordance with Richardson's original model, the remaining parameters $\rho_{1}, \rho_{2}, \sigma_{1}, \sigma_{2}, \epsilon_{1}$ and $\epsilon_{2}$ are set to be nonnegative. The parameter $\beta$ is also taken to be nonnegative, to ensure that distance plays a diminishing role in the values of the weighting factors $w_{l j}$ and $v_{j l}$.

It is possible to simplify the model further, this time at no cost to the generalisability of the model, by rescaling the system. Indeed, substituting

$$
t=\frac{1}{\sigma} \hat{t}, \quad \rho_{i}=\sigma \hat{\rho}_{i}, \quad \epsilon_{i}=\sigma \hat{\epsilon}_{i}
$$


into the model eliminates the parameter $\sigma$. Relabelling the parameters by removing hats, and taking into account the other simplifying assumptions above, the model in equation (11) becomes

$$
\begin{aligned}
& \dot{p}_{j}=-p_{j}+\rho_{1} \sum_{l=1}^{M} q_{l} \frac{p_{j} e^{-\beta d\left(\mathbf{x}_{j}, \mathbf{y}_{l}\right)}}{\sum_{j^{\prime}}^{N} p_{j^{\prime}} e^{-\beta d\left(\mathbf{x}_{j^{\prime}}, \mathbf{y}_{l}\right)}}+\frac{\epsilon_{1}}{N} \\
& \dot{q}_{l}=-q_{l}+\rho_{2} \sum_{j=1}^{N} p_{j} \frac{q_{l} e^{-\beta d\left(\mathbf{y}_{l}, \mathbf{x}_{j}\right)}}{\sum_{l^{\prime}}^{M} q_{l^{\prime}} e^{-\beta d\left(\mathbf{y}_{l^{\prime}}, \mathbf{x}_{j}\right)}}+\frac{\epsilon_{2}}{M} .
\end{aligned}
$$

There are five parameters in equation (13) whose effect on the system dynamics requires exploration. $\rho_{1}$ and $\rho_{2}$ are analogous to the action-reaction terms for each adversary in the original Richardson model and are anticipated to play a similar role. That is, as they increase, the system is expected to become more unstable. A similar comparison can be made for $\epsilon_{1}$ and $\epsilon_{2}$, which are external grievance terms, and are anticipated to play a role in the magnitude of resulting solution curves. The parameter $\beta$, however, has no analogy within the original Richardson model. Its inclusion in equation (13) is as a direct result of the spatial disaggregation.

In the sections that follow, the model in equation (13) is considered in a series of idealised scenarios and, later, more complex scenarios, from which important insights are obtained. Initially, the dimension of the dependent variable, given by $N+M$, is minimised, since low dimensional non-linear systems are often the easiest to analyse. To this end, in Section 4.1, the model is first considered with $N+M=3$, which is the lowest dimension of the dependent variable for which the model admits non-trivial spatial disaggregation of conflict dynamics ( $N=M=1$ leads to Richardson's original system). In Section 4.2, a scenario is considered with $N+M=4$, and then, in Section 4.3, $N+M=8$. In Section 4.4 the model is investigated in a large number of dimensions, using the findings of the more simple scenarios to instruct the analysis.

\subsection{A three-dimensional scenario}

The first scenario to be considered is the simplest with non-trivial spatial disaggregation. Without loss of generality, this is given by the case when $N=2$ and $M=1$, so that one adversary is distributed over two locations - at positions $\mathbf{x}_{1}, \mathbf{x}_{2} \in \mathcal{M}$-and the other adversary remains at just one location, given by $\mathbf{y}_{1} \in \mathcal{M}$. This scenario can be thought of as one step below a macro-level model in which the spatial dependence is 
completely aggregated (and therefore given by the original Richardson model).

In order to fully specify the model, the metric $d$ is defined. A metric is required that distinguishes between the locations $\mathbf{x}_{1}$ and $\mathbf{x}_{2}$, and, for analytic simplicity, is set here so that

$$
d\left(\mathbf{x}_{1}, \mathbf{y}_{1}\right)=0, \quad d\left(\mathbf{x}_{2}, \mathbf{y}_{1}\right)=1
$$

so that the distance between $\mathbf{x}_{1}$ and $\mathbf{y}_{1}$ is negligible, whilst $\mathbf{y}_{1}$ and $\mathbf{x}_{2}$ are different locations on $\mathcal{M}$. The resulting three-dimensional system can be written as

$$
\begin{aligned}
& \dot{p_{1}}=-p_{1}+\rho_{1} \frac{q_{1} p_{1}}{p_{1}+p_{2} e^{-\beta}}+\frac{\epsilon_{1}}{2} \\
& \dot{p_{2}}=-p_{2}+\rho_{1} \frac{q_{1} p_{2} e^{-\beta}}{p_{1}+p_{2} e^{-\beta}}+\frac{\epsilon_{1}}{2} \\
& \dot{q_{1}}=-q_{1}+\rho_{2}\left(p_{1}+p_{2}\right)+\epsilon_{2} .
\end{aligned}
$$

Equation (3) ensures that the dynamics of the variables $p=p_{1}+p_{2}$ and $q=q_{1}$ are given by equation (1) and, consequently, linear stability analysis of the original Richardson model can be utilised. The aggregated system converges to a stable equilibrium if, and only if,

$$
\rho_{1} \rho_{2}<1
$$

(which is consistent with the corresponding condition for equation (1) since the parameters $\sigma_{1} \sigma_{2}$ have been rescaled to one) and this equilibrium is given by

$$
p=p_{1}+p_{2}=\frac{\epsilon_{1}+\rho_{1} \epsilon_{2}}{1-\rho_{1} \rho_{2}}, \quad q=q_{1}=\frac{\epsilon_{2}+\rho_{2} \epsilon_{1}}{1-\rho_{1} \rho_{2}} .
$$

Equation (16) defines a line in three-dimensional $\left(p_{1}, p_{2}, q_{1}\right)$-space as the intersection of two planes. If the stability criteria are satisfied then the system converges to this line. If $\rho_{1} \rho_{2}>1$, then the aggregated system is unstable and almost all solution curves diverge to infinity. For the remainder of this section, it is assumed that $\rho_{1} \rho_{2}<1$, so that all solution curves in the aggregated system converge to a stable equilibrium, and all solution curves in the three-dimensional system in equation (14) converge to the line defined by equation (16). 
It remains to find the dynamics of the system on this line, representing the behaviour of the system that is due to spatial disaggregation. This can be found through a change of variables which separates the model into two components: the original linear Richardson system, which is well-understood, and the unknown dynamics brought about by spatial disaggregation. To this end, the variables

$$
p=p_{1}+p_{2}, \quad r=p_{1}-p_{2},
$$

are introduced. Re-writing the system in equation (14) in terms of the variables $p, q$ and $r$, leads to

$$
\begin{aligned}
& \dot{p}=-p+\rho_{1} q+\epsilon_{1} \\
& \dot{q}=-q+\rho_{2} p+\epsilon_{2} \\
& \dot{r}=-r+\rho_{1} q \frac{p\left(1-e^{-\beta}\right)+r\left(1+e^{-\beta}\right)}{p\left(1+e^{-\beta}\right)+r\left(1-e^{-\beta}\right)},
\end{aligned}
$$

which isolates the unexplored dynamics on the line defined by equation (16) from those of the overall aggregated system. Equations (17) and (18) correspond to the Richardson model in equation (1) with $\sigma_{1}=\sigma_{2}=1$, and do not depend on $r$. The unexplored dynamics captured by equation (19), which encapsulates the effect of spatial disaggregation, can be considered as a distinct system for given values of $p$ and $q$. This system is undefined when

$$
r=-\left(\frac{1+e^{-\beta}}{1-e^{-\beta}}\right) p
$$

and so the analysis presented here is restricted to cases in which this condition does not occur. Indeed, for equality in equation (20), $r$ and $p$ must have opposite signs; however, since $d\left(\mathbf{x}_{1}, \mathbf{y}_{1}\right)<d\left(\mathbf{x}_{2}, \mathbf{y}_{1}\right)$, and since distance is hypothesised to have a diminishing effect on the resulting hostility, it may be assumed that $p_{1}>p_{2}$ for $p>0$ and, therefore, that $r>0$. Thus, this condition is assumed not to occur in scenarios of interest.

There are two real equilibrium points to the one-dimensional system defined by equation (19), given by

$$
r_{ \pm}=-\frac{1}{2}\left(\frac{1+e^{-\beta}}{1-e^{-\beta}}\right)\left(p-\rho_{1} q\right) \pm \frac{1}{2} \sqrt{\left(\frac{1+e^{-\beta}}{1-e^{-\beta}}\right)^{2}\left(p-\rho_{1} q\right)^{2}+4 \rho_{1} p q}
$$

Since $4 \rho_{1} p q>0, r_{-}<0$ and $r_{+}>0$ and thus $r_{+}$is a unique positive equilibrium. 
The local stability of $r_{+}$in the one-dimensional system defined by equation (19) (whilst treating $p$ and $q$ as constants) depends on the sign of

$$
\frac{d \dot{r}}{d r}=-1+\rho_{1} p q \frac{\left(1+e^{-\beta}\right)^{2}-\left(1-e^{-\beta}\right)^{2}}{\left(p\left(1+e^{-\beta}\right)+r\left(1-e^{-\beta}\right)\right)^{2}},
$$

at $r=r_{+}$. It can be shown via algebraic manipulation that

$$
\left.\frac{d \dot{r}}{d r}\right|_{r_{+}}<0
$$

and, therefore, that the positive equilibrium is locally attractive. Furthermore, since the only other equilibrium at $r_{-}<0$ is the only point at which $\dot{r}$ can change sign, $r_{+}$is an attractive equilibrium for $r>0$.

Equation (21) enables the investigation of how the parameters influence the value of $r_{+}$, and therefore influence the resulting spatial distribution of hostility. The parameters $\rho_{1}, \rho_{2}, \epsilon_{1}$ and $\epsilon_{2}$ have a similar interpretation on the aggregate equilibrium value given in equation (16); however, the parameter $\beta$ does not appear in the original model as it results from the spatial disaggregation. Analytically, it can be shown from equation (21) that

$$
\lim _{\beta \rightarrow 0} r_{+}=0, \quad \lim _{\beta \rightarrow \infty} r_{+}=\rho_{1} q
$$

The value of $r_{+}$in equation (21) is plotted in Figure 1 against $\beta$ with the values of the other parameters as given. The parameter $\beta$ determines the extent to which hostility is distributed over the locations $\mathbf{x}_{1}$ and $\mathbf{x}_{2}$, and therefore captures the strength of the spatial dependence in the system. As $\beta \rightarrow 0$, the system becomes aggregated, regardless of the spatial distribution, and the hostility levels in the two locations are equal. As $\beta \rightarrow \infty, r_{+}$reaches its maximum and the system becomes increasingly local: each location interacts only with their closest adversary. The value of $\beta$ specifies the accessibility of the space, and will require appropriate calibration in the application of the model to conflict scenarios.

\subsection{A four-dimensional scenario}

In this section, the complexity of the model is increased by considering a scenario in which each adversary is located over two distinct locations. Suppose that the locations $\mathbf{x}_{1}, \mathbf{x}_{2}, \mathbf{y}_{1}, \mathbf{y}_{2} \in \mathcal{M}$ are associated with 


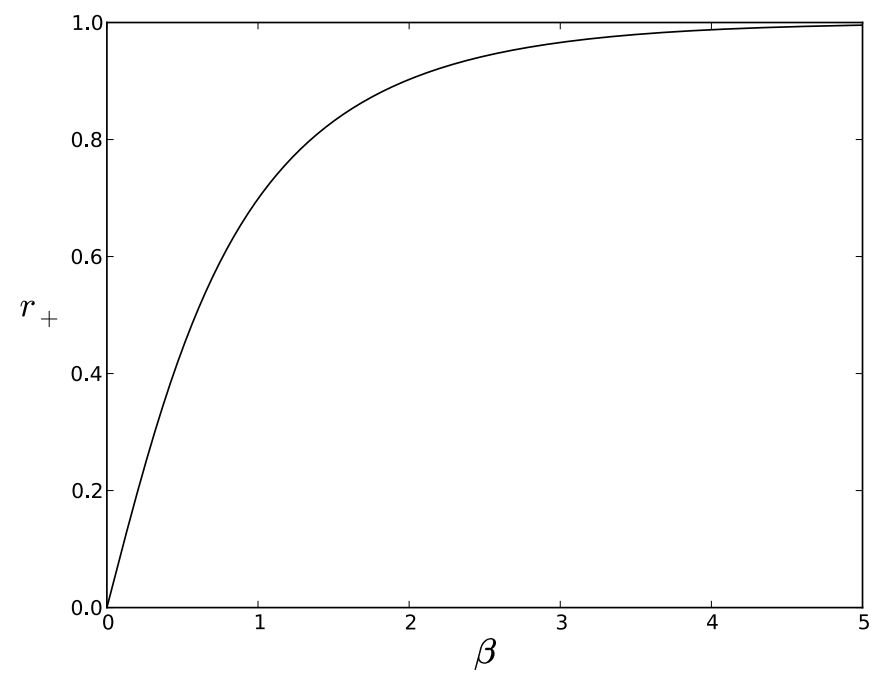

Figure 1: The value of $r_{+}$for different values of $\beta$. The parameter values used are $\epsilon_{1}=\epsilon_{2}=1, \rho_{1}=\rho_{2}=0.5$.

hostility measures $p_{1}, p_{2}, q_{1}, q_{2} \in \mathbb{R}$, respectively. For analytic simplicity, the distance metric $d$ is chosen to consist of zeros and ones. In this case, the $2 \times 2$ matrix $D$ given by $D_{j l}=d\left(\mathbf{x}_{j}, \mathbf{y}_{l}\right)$ is defined to be

$$
D=\left(\begin{array}{ll}
0 & 1 \\
1 & 0
\end{array}\right),
$$

so that adversaries are distributed identically on $\mathcal{M}$. The two adversaries can be thought of as being both distributed across two spatial zones where within-zonal impedance is negligible. In this scenario, the model in equation (13) becomes

$$
\begin{aligned}
& \dot{p_{1}}=-p_{1}+\rho_{1} \frac{q_{1} p_{1}}{p_{1}+p_{2} e^{-\beta}}+\rho_{1} \frac{q_{2} p_{1} e^{-\beta}}{p_{1} e^{-\beta}+p_{2}}+\frac{\epsilon_{1}}{2} \\
& \dot{p_{2}}=-p_{2}+\rho_{1} \frac{q_{1} p_{2} e^{-\beta}}{p_{1}+p_{2} e^{-\beta}}+\rho_{1} \frac{q_{2} p_{2}}{p_{1} e^{-\beta}+p_{2}}+\frac{\epsilon_{1}}{2} \\
& \dot{q_{1}}=-q_{1}+\rho_{2} \frac{p_{1} q_{1}}{q_{1}+q_{2} e^{-\beta}}+\rho_{2} \frac{p_{2} q_{1} e^{-\beta}}{q_{1} e^{-\beta}+q_{2}}+\frac{\epsilon_{2}}{2} \\
& \dot{q_{2}}=-q_{2}+\rho_{2} \frac{p_{1} q_{2} e^{-\beta}}{q_{1}+q_{2} e^{-\beta}}+\rho_{2} \frac{p_{2} q_{2}}{q_{1} e^{-\beta}+q_{2}}+\frac{\epsilon_{2}}{2} .
\end{aligned}
$$

Similarly to the three dimensional case, the dynamics of the original Richardson model can be extracted from this system by a change of variables, leading to a reduced dynamical system to which the system converges 
for $\rho_{1} \rho_{2}<1$. The following variables are therefore introduced:

$$
\begin{array}{ll}
p=p_{1}+p_{2}, & r=p_{1}-p_{2}, \\
q=q_{1}+q_{2}, & s=q_{1}-q_{2} .
\end{array}
$$

Substituting these expressions into equation (23), and re-writing the system so that it depends only on $p, q$, $r$ and $s$, obtains

$$
\begin{aligned}
\dot{p}= & -p+\rho_{1} q+\epsilon_{1} \\
\dot{q}= & -q+\rho_{2} p+\epsilon_{2} \\
\dot{r}= & -r+\frac{\rho_{1}}{2}(q+s) \frac{\left(1-e^{-\beta}\right) p+\left(1+e^{-\beta}\right) r}{\left(1+e^{-\beta}\right) p+\left(1-e^{-\beta}\right) r} \\
& +\frac{\rho_{1}}{2}(q-s) \frac{\left(e^{-\beta}-1\right) p+\left(1+e^{-\beta}\right) r}{\left(1+e^{-\beta}\right) p+\left(e^{-\beta}-1\right) r} \\
\dot{s}= & -s+\frac{\rho_{2}}{2}(p+r) \frac{\left(1-e^{-\beta}\right) q+\left(1+e^{-\beta}\right) s}{\left(1+e^{-\beta}\right) q+\left(1-e^{-\beta}\right) s} \\
& +\frac{\rho_{2}}{2}(p-r) \frac{\left(e^{-\beta}-1\right) q+\left(1+e^{-\beta}\right) s}{\left(1+e^{-\beta}\right) q+\left(e^{-\beta}-1\right) s} .
\end{aligned}
$$

Equations (24) and (25) are equivalent to the original Richardson system with $\sigma_{1}=\sigma_{2}=1$, whilst equations (26) and (27) represent the added dynamics and complexity that is due to spatial disaggregation. For $\rho_{1} \rho_{2}<1$, the system converges to the plane defined by the equilibrium of the aggregated system, given by

$$
p=\frac{\rho_{1} \epsilon_{2}+\epsilon_{1}}{1-\rho_{1} \rho_{2}}, \quad q=\frac{\rho_{2} \epsilon_{1}+\epsilon_{2}}{1-\rho_{1} \rho_{2}} .
$$

For the remainder of the section, it is assumed that $\rho_{1} \rho_{2}<1$ and that a sufficient amount of time has passed so that $p$ and $q$ have reached their equilibrium values, and the unexplored dynamics of the system are given by equations (26) and (27), where $p$ and $q$ are constants given in equation (28).

The system is undefined when

$$
r= \pm\left(\frac{1+e^{-\beta}}{1-e^{-\beta}}\right) p,
$$

or when

$$
s= \pm\left(\frac{1+e^{-\beta}}{1-e^{-\beta}}\right) q .
$$


Consequently, the analysis presented here is restricted to solutions that do not cross this region in phase space. For a value of $\beta>0$, the lines at which the system is undefined generate a rectangle in $r s$-space surrounding the origin. Considering possible solutions within this rectangle, it can be observed that the origin is an equilibrium, representing the point at which $p_{1}=p_{2}$ and $q_{1}=q_{2}$ and thus where hostility is equally distributed amongst the different locations in space.

Linearisation of the planar system in equations (26) and (27) about the origin leads to

$$
\left(\begin{array}{c}
\dot{r} \\
\dot{s}
\end{array}\right)=\left(\begin{array}{cc}
-1+\rho_{1}\left(1-\eta^{2}\right) & \rho_{1} \eta \\
\rho_{2} \eta & -1+\rho_{2}\left(1-\eta^{2}\right)
\end{array}\right)\left(\begin{array}{c}
r \\
s
\end{array}\right),
$$

where

$$
\eta=\left(\frac{1-e^{-\beta}}{1+e^{-\beta}}\right)
$$

which is dependent on $\beta>0$ in such a way so that $0<\eta<1$. The eigenvalues of the system in equation (29) are

$$
\lambda_{ \pm}=-1+\frac{1}{2}\left(\rho_{1}+\rho_{2}\right)\left(1-\eta^{2}\right) \pm \frac{1}{2} \sqrt{\left(\rho_{1}-\rho_{2}\right)^{2}\left(1-\eta^{2}\right)^{2}+4 \eta^{2} \rho_{1} \rho_{2}} .
$$

For clarity, a simplified scenario in which $\rho_{1}=\rho_{2}=\rho$ is first considered. This implies that the intensity of the action-reaction dynamics for each adversary is equal. The eigenvalues simplify to

$$
\lambda_{ \pm}=-1+\rho\left(1-\eta^{2} \pm \eta\right)
$$

Considering first $\lambda_{-}$,

$$
\lambda_{-}=-1+\rho\left(1-\eta^{2}-\eta\right)<-1+\rho<0
$$

since $\eta>0$ and $0<\rho<1$. Thus one eigenvalue is always negative and the condition for stability depends solely on the eigenvalue $\lambda_{+}$. In particular, the equilibrium is stable when

$$
\lambda_{+}=-1+\rho\left(1-\eta^{2}+\eta\right)<0
$$

which occurs when

$$
\rho<\frac{1}{1-\eta^{2}+\eta}=\frac{\left(e^{\beta}+1\right)^{2}}{e^{2 \beta}+4 e^{\beta}-1} .
$$


Considering the right hand side of equation (35),

$$
0<\frac{\left(e^{\beta}+1\right)^{2}}{e^{2 \beta}+4 e^{\beta}-1}<\frac{e^{2 \beta}+2 e^{\beta}+1+2\left(e^{\beta}-1\right)}{e^{2 \beta}+4 e^{\beta}-1}=1,
$$

and, thus, for $\rho<1$, it is possible that the equilibrium can be either stable or unstable, depending on the value of $\rho$ in comparison to the value $\bar{\rho}$ given by

$$
\bar{\rho}(\beta)=\frac{\left(e^{\beta}+1\right)^{2}}{e^{2 \beta}+4 e^{\beta}-1} .
$$

A bifurcation occurs as $\rho$ increases above $\bar{\rho}$, and $\bar{\rho}$ is said to be a bifurcation point.

In Figure 2, the function $\bar{\rho}(\beta)$ for $\beta>0$ is shown, in order to demonstrate how the bifurcation point $\bar{\rho}$ varies with the parameter $\beta$. The minimum of $\bar{\rho}$ occurs at

$$
\beta_{\min }=\ln 3 \approx 1.1, \quad \bar{\rho}\left(\beta_{\min }\right) \approx 0.8
$$

The existence of the bifurcation has important implications for the model. Given an appropriate value for $\beta$,

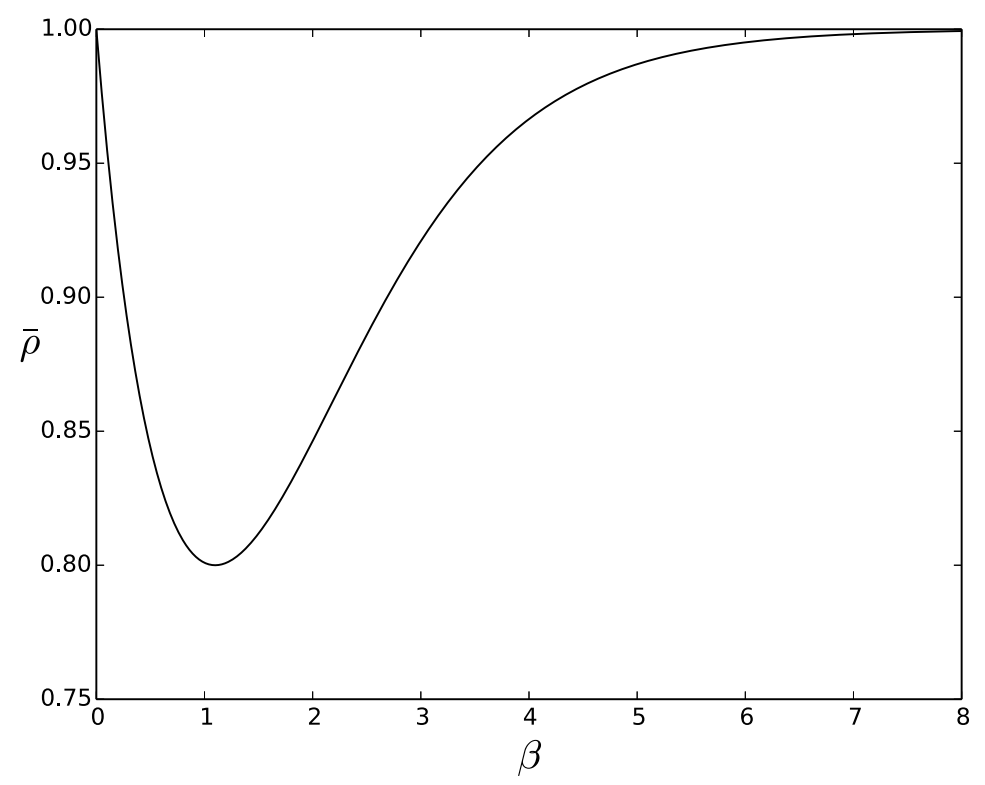

Figure 2: The bifurcation point $\bar{\rho}$ plotted against $\beta$.

for relatively small values of $\rho<1$, corresponding to scenarios in which retaliatory dynamics are weak, then hostile activity is likely to be evenly distributed in space. However, if $\rho<1$ is close to one, corresponding to 
scenarios with stronger retaliation and therefore higher levels of aggression, then hostile activity is likely to be more unevenly distributed, even if the aggregate system converges to a stable equilibrium.

Taking the Taylor expansion of the planar system given by equations (26) and (27) about the origin, and retaining terms up to third order, leads to

$$
\left(\begin{array}{c}
\dot{r} \\
\dot{s}
\end{array}\right)=\left(\begin{array}{c}
\left(-1+\rho-\rho \eta^{2}\right) r+\rho \eta s+\frac{\rho \eta^{2}}{p^{2}}\left(1-\eta^{2}\right) r^{3}-\frac{\rho \eta}{p^{2}}\left(1-\eta^{2}\right) r^{2} s \\
\left(-1+\rho-\rho \eta^{2}\right) s+\rho \eta r+\frac{\rho \eta^{2}}{q^{2}}\left(1-\eta^{2}\right) s^{3}-\frac{\rho \eta}{q^{2}}\left(1-\eta^{2}\right) s^{2} r
\end{array}\right),
$$

which can be used to identify the existence of two stable equilibria for $\rho>\bar{\rho}$, some solution curves of which are shown in Figure 3 for $\rho<\bar{\rho}$ and $\rho>\bar{\rho}$.
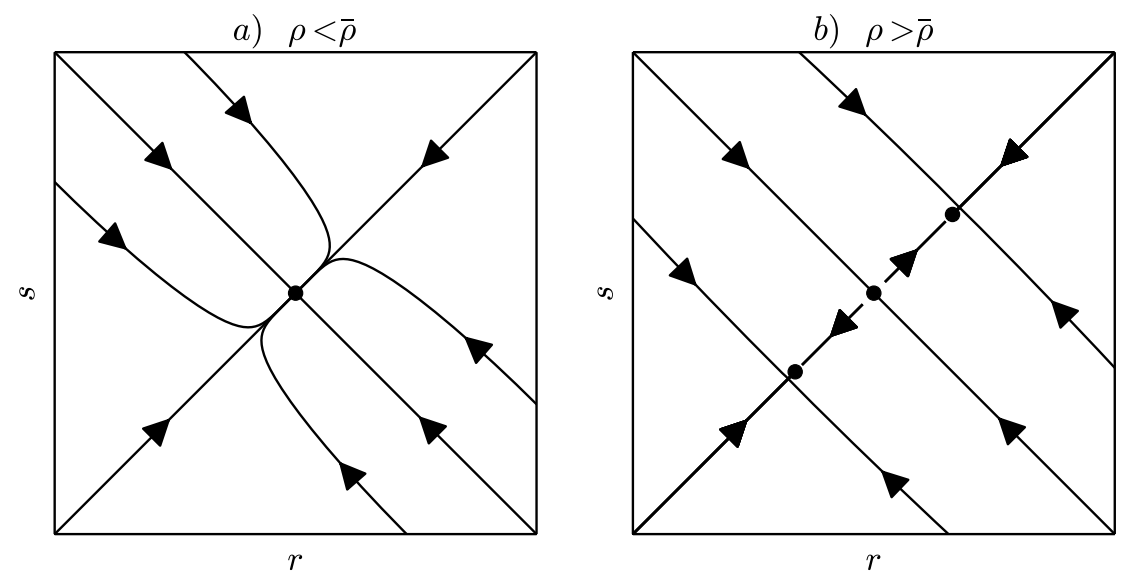

Figure 3: Selected solution curves of the system in equation (39) for two different values of $\rho$. In a), $\rho=0.7$, whilst in b), $\rho=0.803$. All other parameter values are such that $\bar{\rho}=0.801$.

Supposing that $r=s$ and $\epsilon_{1}=\epsilon_{2}$ in equation (39), leads to

$$
\dot{r}=\left(-1+\rho\left(1-\eta^{2}+\eta\right)\right) r-\left(\frac{\rho \eta}{p^{2}}\left(1-\eta^{2}\right)(1-\eta)\right) r^{3}
$$

If $\epsilon_{1}=\epsilon_{2}$, then the system in equation (39) is symmetric and equilibria of the system in equation (40) will correspond to equilibria of the system in equation (39). Equilibria of the system in equation (40) occur when either $r=0$ or when

$$
r^{2}=\frac{1-\rho\left(1-\eta^{2}+\eta\right)}{\frac{\rho \eta}{p^{2}}\left(1-\eta^{2}\right)(\eta-1)}
$$


which has solutions only when the right-hand side of equation (41) is greater than zero. It can be shown that this occurs when

$$
\rho>\frac{1}{1-\eta^{2}+\eta}
$$

corresponding to the condition for the loss of stability in the equilibrium at the origin. In Figure 4, the equilibrium values for the system in (40) are shown for different values of $\rho$. As $\rho$ increases beyond $\bar{\rho}$, two stable equilibria appear, with values given by

$$
r_{ \pm}= \pm p\left(\frac{1-\rho\left(1-\eta^{2}+\eta\right)}{\rho \eta\left(1-\eta^{2}\right)(\eta-1)}\right)^{\frac{1}{2}}
$$

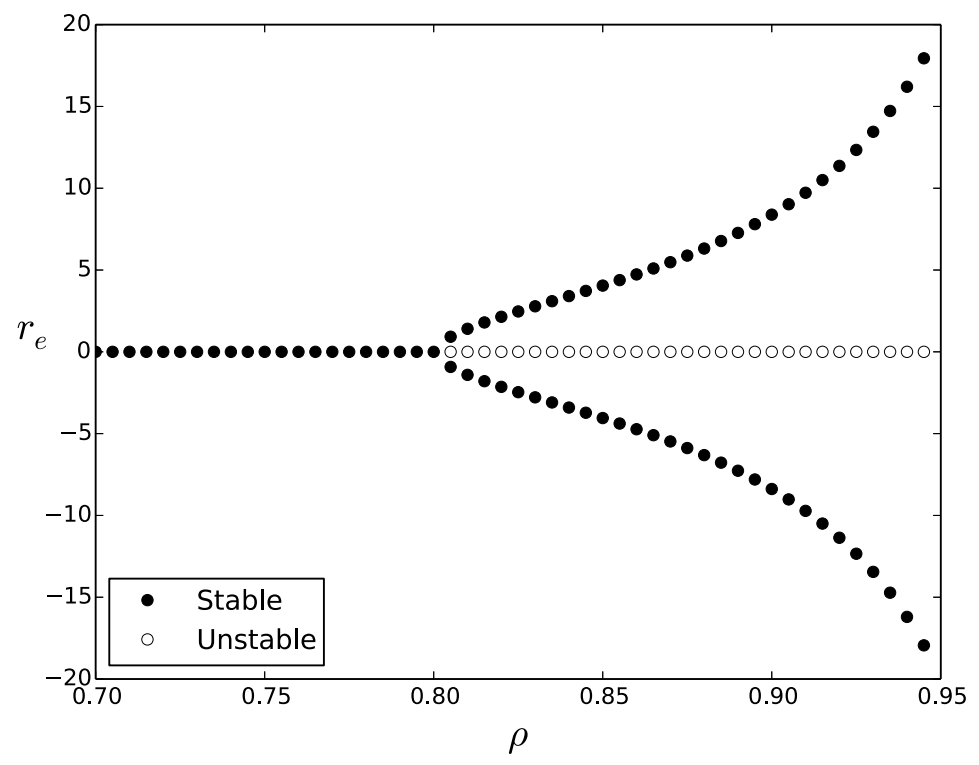

Figure 4: Equilibria of equation (40) for varying values of $\rho$.

A bifurcation of the system in equations (26) and (27) at the equilibrium of the $r$ s-plane has been shown to exist in the special case when $\rho_{1}=\rho_{2}$. It is important to determine whether the same bifurcation occurs when $\rho_{1} \neq \rho_{2}$. This is because conflict scenarios to which the model may be applied will often be asymmetric: each adversary may adopt different tactics, resulting in different retaliatory mechanisms and therefore result in different action-reaction parameters, as given by $\rho_{1}$ and $\rho_{2}$. In Figure 5 the stability of the origin of the 
$r s$-plane is shown for values of $\rho_{1}$ and $\rho_{2}$ between 0.5 and 1 , and for three different values of $\beta$. In this figure, green represents stability of the equilibrium, and blue represents instability. The bifurcation can be observed in the transition from stability to instability in each of the three cases considered, across different values for $\beta$. This figure confirms that the identified bifurcation is persistent under variation of the parameter values. Furthermore, the change in the bifurcation point appears to be smooth with varying parameters: an increase in $\rho_{1}$ moves the bifurcation point in the direction of decreasing $\rho_{2}$. This suggests that the system requires some total sum of aggression, as determined by a combination of the parameters $\rho_{1}$ and $\rho_{2}$, before the equilibrium at the origin of the $r s$-plane becomes unstable.
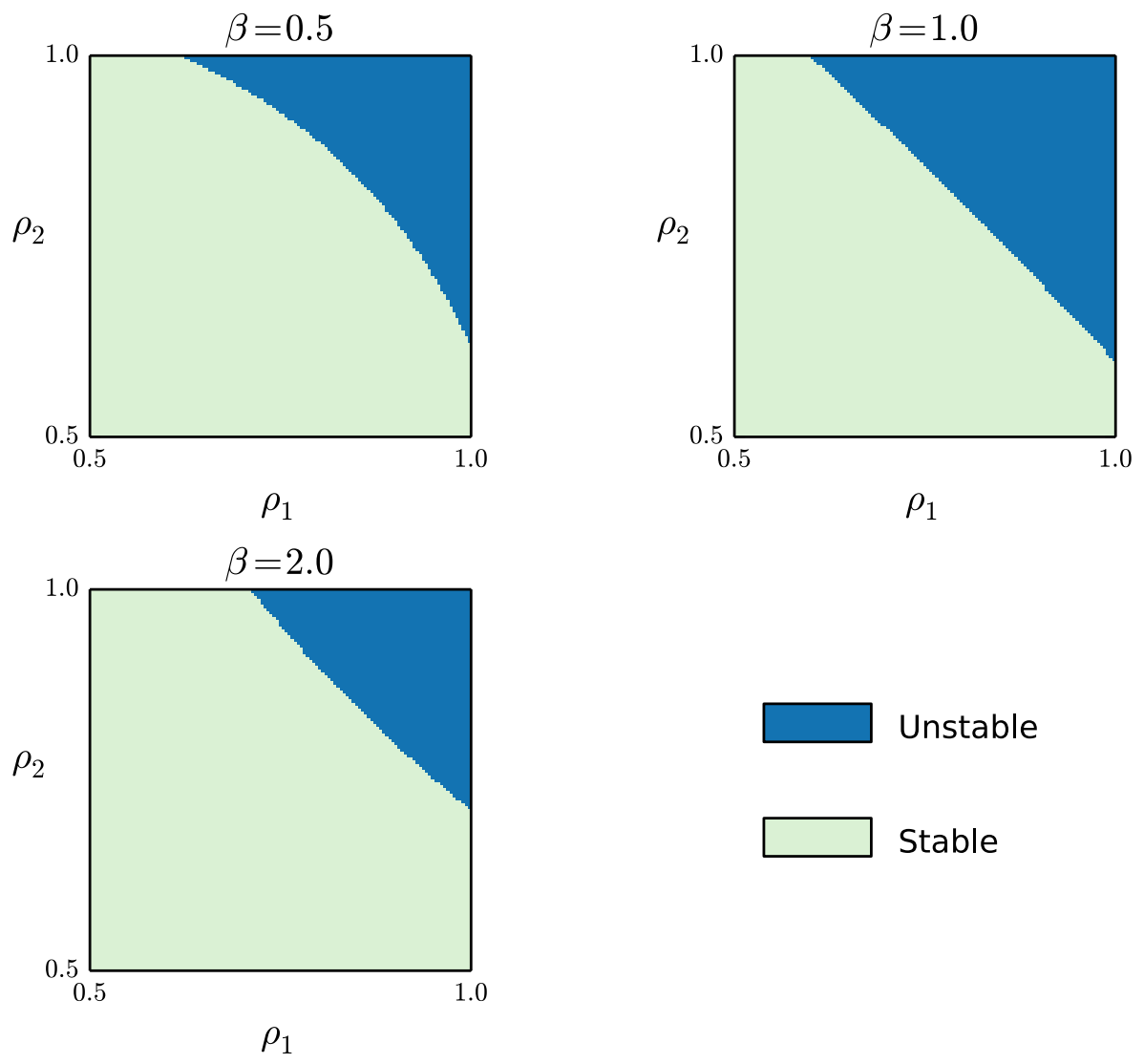

Figure 5: Stability of the equilibrium at the origin of the $r s$-plane for $\rho_{1} \in[0.5,1)$ and $\rho_{2} \in[0.5,1)$ and for $\beta=0.5, \beta=1$, and $\beta=2$.

The four-dimensional model given in equation (23) has been shown to exhibit richer behaviour than the 
three-dimensional case. By investigating the stability of the most natural equilibrium point in the system, given by the point at which hostility levels are equally distributed over adversaries, a supercritical pitchfork bifurcation has been identified in the case of a symmetric system, which can occur within a feasible region of the parameter space. It has been shown that a bifurcation persists for asymmetric conflicts, which we suspect to be of pitchfork form. In what follows, further properties of the bifurcation are sought that serve to remove any suspicions of reliance on some of the limiting assumptions employed in this section. In particular, the model is considered with different distance metrics and in higher-dimensional scenarios.

\subsection{An eight-dimensional scenario}

The bifurcation identified in Section 4.2 may have existed as a consequence of the particular form of the distance metric or as a result of the number of dimensions used. In this section, two eight-dimensional scenarios with $N=M=4$ are explored, and the stability of the most natural equilibrium as system-wide aggression increases is investigated. Doubling the dimension of the model leads to a reduction in analytical tractability. As a consequence, numerical simulation of the model is used to explore the range of potential scenarios in what follows. Numerical simulations are performed using the Runge-Kutta method for temporal discretisation. It was found that step sizes of 0.1 produced simulation results in agreement with much smaller step sizes as well as in agreement with the analytical results presented in the previous sections.

The two scenarios are constructed by defining the $4 \times 4$ matrices $D^{(1)}$ and $D^{(2)}$ where $D_{j l}^{(i)}=d\left(\mathbf{x}_{j}, \mathbf{y}_{l}\right)$ for $i=1,2$ and are given by

$$
D^{(1)}=\left(\begin{array}{cccc}
0 & 1 & 1 & 1 \\
1 & 0 & 1 & 1 \\
1 & 1 & 0 & 1 \\
1 & 1 & 1 & 0
\end{array}\right), \quad D^{(2)}=\left(\begin{array}{cccc}
0 & 1 & 2 & 3 \\
1 & 0 & 1 & 2 \\
2 & 1 & 0 & 1 \\
3 & 2 & 1 & 0
\end{array}\right) .
$$

$D^{(1)}$ defines a scenario that is a natural extension to the example in Section 4.2. It is assumed that, instead of adversaries being located across two zones, they are instead located across four zones. In this case, impedance within the same zone is negligible, whilst any two distinct zones are a significant distance 
from each other. $D^{(2)}$ defines a scenario in which a different spatial structure is imposed on the system. In this case, since the distance between $j$ and $l$ is determined by $|j-l|$ for $j, l=1,2,3,4$, consecutive zones can be considered to be near to each other in space, and non-consecutive zones farther apart. In contrast to the previous example, the strength of inter-zone interaction now varies according to the particular pairing in question.

Figure 6(a) shows two runs of the model with distance metric $D^{(1)}$ and Figure 6(b) shows two runs of the model with distance metric $D^{(2)}$. The two different runs of the model are chosen to correspond to two scenarios in which the level of aggression in the system, determined by the parameters $\rho_{1}$ and $\rho_{2}$, lies on either side of some characteristic level of aggression, denoted by $\hat{\rho}$. The value of $\hat{\rho}$ is obtained by utilising the results of the previous section, in which it was found that, when two adversaries with equal aggression have parameters that exceed 0.8 , a bifurcation occurs. For each scenario considered, the sum of the two solution curves in each zone is plotted for each model run and, in both cases, a bifurcation is observed: on either side of the characteristic value of $\hat{\rho}$, a qualitatively different equilibrium is obtained.

In the case of Figure 6(a), prior to the bifurcation, analogously to the scenario studied in Section 4.2, the system converges to an equilibrium which is constant across each zone. Since the aggregated system is given by the original Richardson system, this equilibrium is given by

$$
p_{j}=\frac{\rho_{1} \epsilon_{2}+\epsilon_{1}}{4\left(1-\rho_{1} \rho_{2}\right)}, \quad q_{l}=\frac{\rho_{2} \epsilon_{1}+\epsilon_{2}}{4\left(1-\rho_{1} \rho_{2}\right)},
$$

for $j, l=1,2,3,4$. After the bifurcation occurs, solution curves in zone 1 converge to a larger equilibrium value and solution curves in the other zones decrease to compensate for the increase in zone 1 . In performing both numerical simulations, initial conditions in zone 1 were perturbed slightly to ensure the solution curves did not rest on the potentially unstable equilibrium in equation (44). We conjecture that it is for this reason that hostility levels increase in zone 1, as opposed to any of the other zones.

In Figure 6(b), prior to the bifurcation, the system converges to a natural equilibrium, which, according 

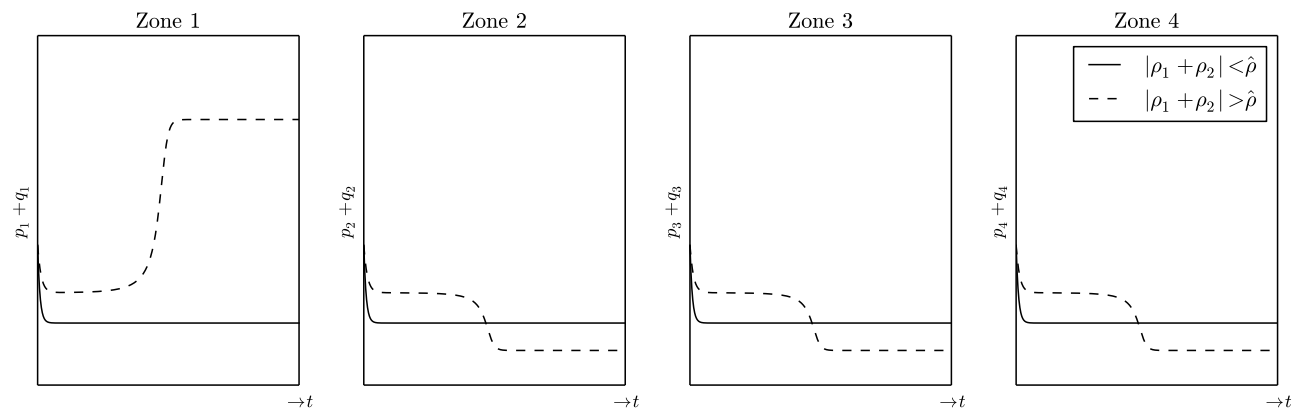

(a) A scenario with distance metric given by $D^{(1)}$.
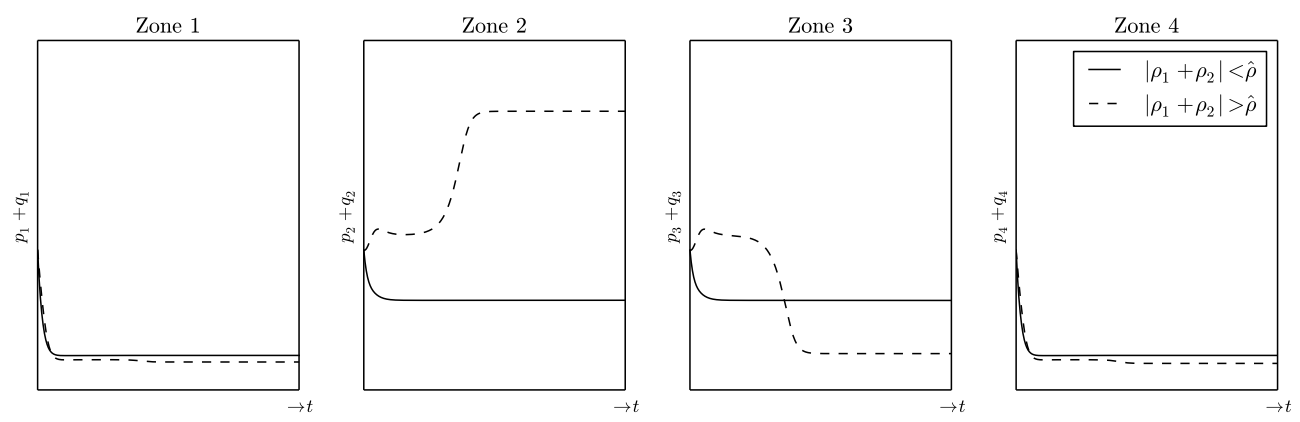

(b) The same scenario but with distance metric given by $D^{(2)}$.

Figure 6: The sum of the two solution curves in each zone, for two different sets of parameter values for scenarios defined by $D^{(1)}$ and $D^{(2)}$ in equation (43). For the two scenarios, the parameters values are, in the case of the solid line, $\rho_{1}=0.75, \rho_{2}=0.8, \epsilon_{1}=\epsilon_{2}=0.4$, and $\beta=1$; and, in the case of the dashed line, $\rho_{1}=0.8, \rho_{2}=0.9, \epsilon_{1}=\epsilon_{2}=0.4$ and $\beta=1$. 
to the numerical simulation, is given by

$$
\begin{array}{llll}
p_{1}=0.21, & p_{2}=0.25, & p_{3}=0.25, & p_{4}=0.21, \\
q_{1}=0.20, & q_{2}=0.23, & q_{3}=0.23, & q_{4}=0.20 .
\end{array}
$$

In this case, the hostility levels are not equal across zones. The relative values of the equilibrium emphasise the spatial structure of the system, since zones 2 and 3 are the zones that are closest to all other zones and will therefore have the greatest interaction with the other zones. The equilibrium brought about by the magnitude of aggression in the system being less than $\hat{\rho}$ is not the equilibrium that leads to equality of resulting hostility across zones, but represents a spatially-weighted equilibrium in which zones that are closest to other zones naturally contain higher levels of hostility than zones that are farther away.

After the bifurcation, a different equilibrium is found and a bifurcation is observed with this different spatial structure. Similarly to the previous example, zone 1 is given perturbed initial conditions to avoid resting on an unstable equilibrium. However, in this case, rather than zone 1 experiencing a dramatic increase in hostility levels, zone 2 is the one that increases. It is hypothesised that this is due to the more central location of zone 2 in comparison to zone 1 .

This section has explored the application of the model to two spatial conflict scenarios. The two eightdimensional models considered have been shown to contain bifurcation-type behaviour for feasible parameter values, supporting the hypothesis that the bifurcation identified in Section 4.2 exists in higher dimensions and in more general spaces. The second example used in this section, in which the spatial structure of the system is less symmetric, has shown that the most natural equilibrium to which the system appears to converge for small values of $\rho_{1}$ and $\rho_{2}$ is not necessarily an equilibrium with equal hostility levels in each zone, as was the case for the first example. Instead, the model appears to converge to a spatially-weighted equilibrium, for which adversaries located closest to other adversaries have a higher resulting level of hostility. The fact that a spatially-weighted solution is an equilibrium confirms intuition regarding the evolution of spatial conflict: it is those areas nearest to an adversary that are likely to experience greater levels of conflict over long periods of time. The existence of the bifurcation, however, and the somewhat unpredictable nature of resulting 
solution curves after the bifurcation has taken place, is a counter-intuitive finding that is generalised further in the next section.

\subsection{A Euclidean large N-dimensional model}

Conflicts can occur over large areas and involve participants in a number of distinct locations. So far, only scenarios involving up to four distinct locations on each side of the conflict have been considered. The model is now investigated in higher dimensions, demonstrating how the model might be scaled up to consider conflict occurring over large spatial scales. We test whether there is evidence that the bifurcation identified in Sections 4.2 and 4.3 exists in these more general scenarios. To this end, the model proposed in this section contains 100 dimensions, in which $N$ is not necessarily equal to $M$, and in which a Euclidean distance metric is used that is distinct from the zonal approach to defining the metrics used previously.

To specify the model, we first set $\mathcal{M}=\left\{(x, y) \in \mathbb{R}^{2} \mid 0 \leq x \leq 1,0 \leq y \leq 1\right\}$, so that adversaries are located within a unit square, and we define $d: \mathcal{M} \times \mathcal{M} \rightarrow \mathbb{R}$ to be the Euclidean distance metric. One hundred points in $\mathcal{M}$ are uniformly randomly generated and each point is uniformly randomly allocated to either one of two adversaries. Each point is assigned an initial hostility level equal to one.

The model is then initialised with $\epsilon=\epsilon_{1} / N=\epsilon_{2} / M=0.1, \beta=1$ and $\rho_{1}=\rho_{2}=\rho$. Solutions of the system in equation (13) are numerically solved using the Runge-Kutta method. If there exists $\bar{t}>0$ such that

$$
\left|p_{j}(\bar{t}+\delta t)-p_{j}(\bar{t})\right|<10^{-3}, \quad\left|q_{l}(\bar{t}+\delta t)-q_{l}(\bar{t})\right|<10^{-3},
$$

for $j=1,2, \ldots, N$ and $l=1,2, \ldots, M$, where $\delta t=0.1$, it is assumed that the system converges to an equilibrium and that this equilibrium is given by the values of $p_{j}(\bar{t})$ and $q_{l}(\bar{t})$ for $j=1,2, \ldots, N$ and $l=1,2, \ldots M$, where $t=\bar{t}$ is the first value of $t$ for which the condition in equation (45) holds.

This process is repeated 100 times, ensuring that a range of random spatial configurations of the model are tested. For each of these, equilibria for different values of $\rho$ are calculated and these are compared using the skewness of the distribution of equilibrium values over the different locations of one of the adversaries. 
That is, for each random spatial configuration $k$ and for each $\rho$, we calculate

$$
\mathcal{S}^{(k)}(\rho)=\frac{\frac{1}{N} \sum_{j=1}^{N}\left(p_{j}^{(k)}(\bar{t} ; \rho)-\bar{p}^{(k)}(\bar{t} ; \rho)\right)^{3}}{\left(\frac{1}{N} \sum_{j=1}^{N}\left(p_{j}^{(k)}(\bar{t} ; \rho)-\bar{p}^{(k)}(\bar{t} ; \rho)\right)^{2}\right)^{3 / 2}}
$$

where $\left.\bar{p}^{(k)}(\bar{t} ; \rho)\right)$ is the mean of the equilibrium values $p_{1}^{(k)}(\bar{t} ; \rho), p_{2}^{(k)}(\bar{t} ; \rho), \ldots, p_{N}^{(k)}(\bar{t} ; \rho)$, for $k=1,2, \ldots, 100$.

Skewness measures the asymmetry in a set of values. If a set of values are positioned evenly on either side of their mean, as in a Gaussian curve, the skew will be zero. If there is a long tail of large values in comparison to the mean then the skew will be positive. Figure 7 shows the distribution of $\mathcal{S}^{(k)}(\rho)$ over $k$ as $\rho$ increases. The solid line represents the mean of the skew statistic over 100 random spatial configurations, the darker shaded region represents the interquartile range (within which $50 \%$ of the values are situated), and the lighter shaded region represents a region containing $90 \%$ of the values of the skew statistic over the different realisations.

Increasing $\rho$ from 0.5 slowly increases the skew statistic from a mean of around zero. As $\rho$ increases further, after around $\rho=0.8$, the value of the skew dramatically increases before settling at a maximum. For small values of $\rho$, the values are therefore largely symmetric around the mean, but, as $\rho$ increases, a small number of increasingly large values start to make a disproportionately large contribution to the value of the mean. This finding is consistent with the loss of stability of a naturally weighted equilibrium as $\rho$ increases, providing evidence that a bifurcation persists in this more general setting.

Figures 7(a) and 7(b) show the modelled scenario for two values of $\rho$, one on each side of a potential bifurcation. The colour of each point is used to distinguish between the adversaries and the position of each point represents its location on $\mathcal{M}$. The size of the point is proportional to the corresponding equilibrium value at that location. As $\rho$ increases, hostility becomes increasing concentrated within a small number of locations. These locations are towards the centre of the manifold; however, it would be difficult to predict these locations a priori, since there are many possible locations that might have experienced a similar increase in hostility. The system with $\rho=0.9$ might be considered much more unpredictable and potentially more dangerous than the system with $\rho=0.8$, in which the hostility levels are more balanced over the possible locations. That is, we get a change from broadly-spread tension to an acute concentration of hostility levels 


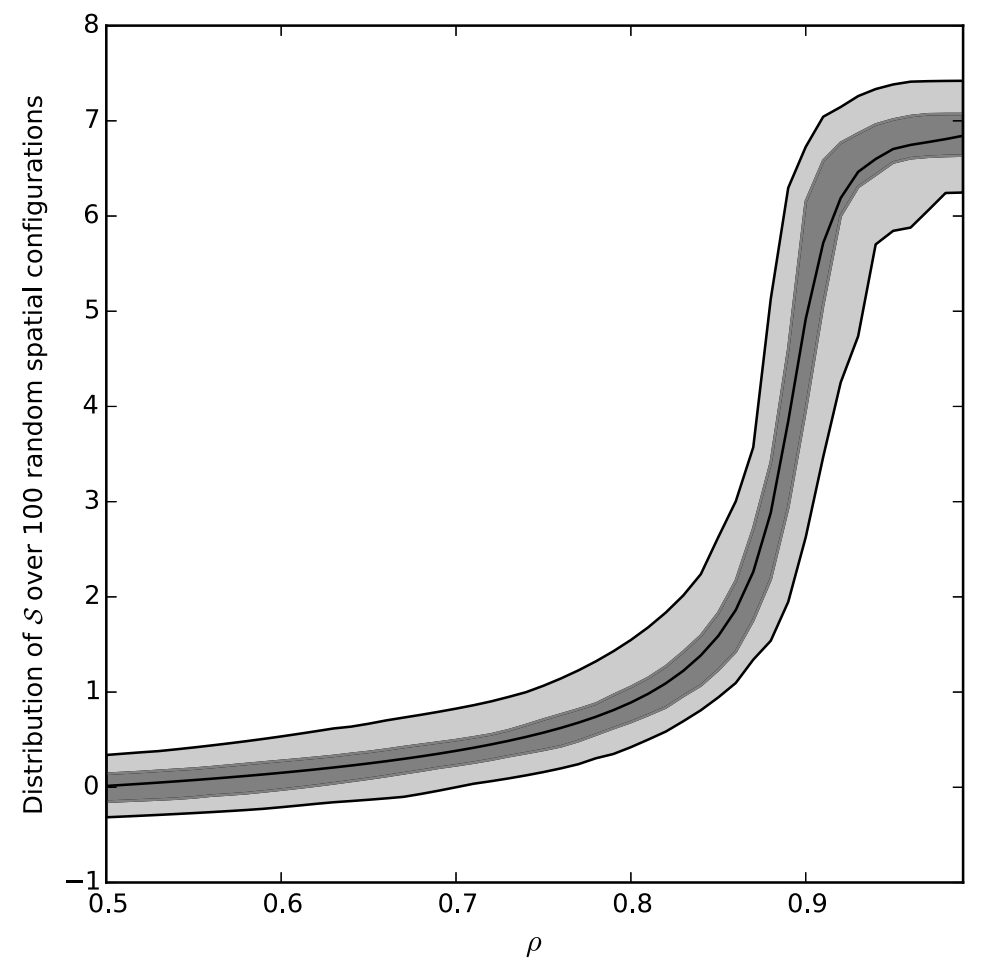

a) $\rho=0.8$

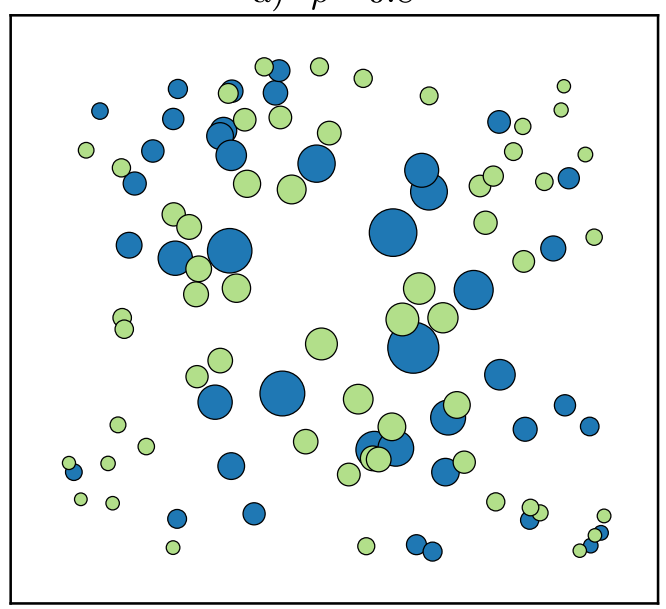

b) $\rho=0.9$

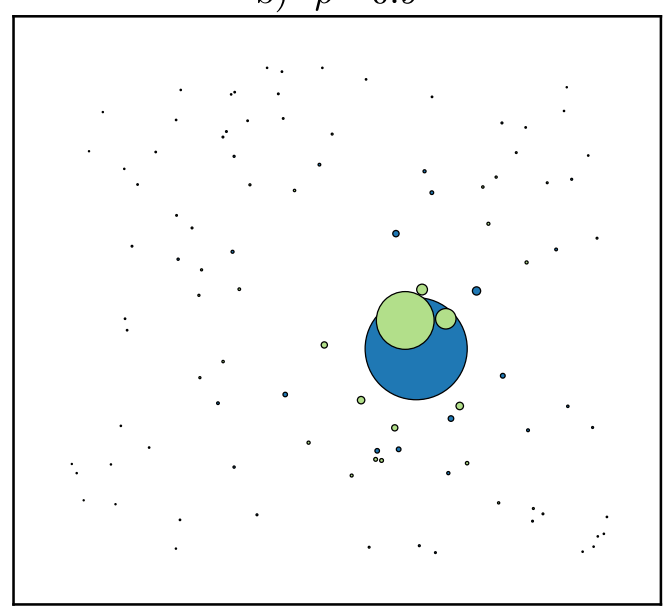

Figure 7: The distribution of the skew statistic $\mathcal{S}^{(k)}(\rho)$ over 100 random spatial configurations of the model for different values of $\rho$. Subfigures a) and b) show the equilibrium of one spatial configuration for two different values of $\rho$. 
in a very small area. In the latter case, the system might be interpreted as being on the brink of a severe escalation in hostility.

Evidence has been presented that the bifurcation identified in Section 4.2 is persistent under a variety of parameter values, dimensions and distance metrics. This is a significant result brought about by the spatial disaggregation of the system, and highlights the types of insights that can be obtained using non-linear dynamical systems analysis. In particular, according to the model, in spatially dependent systems with increasing aggression, a qualitative change in the spatial distribution of hostility levels would be anticipated to be observed, before the entire system becomes unstable and an arms race is initiated (according to the original Richardson model).

\section{Conclusion}

We have proposed a novel method for the spatial disaggregation of models of conflict by considering how an abstract notion of 'threat' flows between two adversaries. We have demonstrated this approach by spatially disaggregating the Richardson model of conflict escalation between two adversaries, and explored the resulting spatial dependency. A bifurcation was identified that occurs as the magnitude of aggression in the system increases, and was shown to be persistent under a range of model specifications.

Prior to the bifurcation, for low levels of aggression in the system, solution curves are expected to converge naturally to an equilibrium which is spatially weighted according to the relative locations of adversaries. For higher levels of aggression in the system, once the bifurcation has occurred, the spatially weighted equilibrium becomes unstable and the model converges to a new equilibrium in which hostility levels are highly concentrated within a few locations. Increasing the level of aggression in the system further can, as demonstrated in the analysis of Richardson's original model, lead to an unstable escalating arms race. The bifurcation hints at a potential early-warning system for real-world conflicts: with increasing aggression, before the system results in an arms race and hostility increases exponentially, some spatial instability is expected and particular locations may suddenly experience disproportionate increases in hostility or conflict. 
If vulnerable locations can be identified prior to such increases in aggression, then policy interventions might seek to reduce tensions in those areas that are likely to experience this initial increase in hostility.

There are a number of avenues for further research. First, the model has been explored within a relatively restricted region of the phase space. Specifically, it was the geographically weighted equilibrium, which was attractive for low levels of aggregation in the system, and the deviation from this equilibrium as the level of aggression increased, that was explored. Since the model is nonlinear, a number of other trajectories and equilibrium solutions are possible. The analysis was also not extensive with respect to the range of parameters considered and thus further research might do more to comprehensively explore the parameter space. Further investigation into the nature of the bifurcation - for example, via a centre manifold reduction that employs equivariant bifurcation theory or via parameter-following computational techniques-might lead to a greater understanding as to its emergence and persistence. Finally, the model has not yet been applied to real-world scenarios in order to assess its empirical validity. Calibration of the model against empirical data might determine where the system lies in the phase space, and therefore might indicate whether the system is near to an undesirable bifurcation.

The analysis presented here is a significant contribution to the literature exploring spatial dependency in models of human conflict. We have presented an alternative method based on the principle of maximum entropy for capturing such dependency, which alleviates some of the problems that might be encountered when employing reaction-diffusion dynamics. In addition, the spatial disaggregation of the Richardson model provides an analytical framework to explore a range of conflict scenarios that play out over some manifold with an associated metric. The identification of a bifurcation that occurs as a result of this disaggregation highlights the complex dynamics that the model is capable of exhibiting, as well as providing potential insights into how the spatial distribution of conflict evolves in systems with increasing aggression. 


\section{Acknowledgements}

The authors acknowledge the financial support of the Engineering and Physical Sciences Research Council (EPSRC) under the grant ENFOLD-ing - Explaining, Modelling and Forecasting Global Dynamics, reference EP/H02185X/1. We also thank two anonymous reviewers whose comments have helped improve this manuscript.

\section{References}

Atkinson, M. P., Gutfraind, A., and Kress, M. 2011. When do armed revolts succeed: Lessons from Lanchester theory. Journal of the Operational Research Society, 63(10):1363-1373.

Blank, L., Enomoto, C. E., Gegax, D., Mcguckin, T., and Simmons, C. 2008. A dynamic model of insurgency: The case of the war in Iraq. Peace Economics, Peace Science and Public Policy, 14(2):1-26.

Brantingham, P. J., Tita, G. E., Short, M. B., and Reid, S. E. 2012. The ecology of gang territorial boundaries. Criminology, 50(3):851-885.

Davies, T. P., Fry, H. M., Wilson, A. G., and Bishop, S. R. 2013. A mathematical model of the London riots and their policing. Scientific reports, 3:1303.

Deitchman, S. J. 1962. A Lanchester model of guerrilla warfare. Operations Research, 10(6):818-827.

Dennett, A. and Wilson, A. G. 2013. A multi-level spatial interaction modelling framework for estimating inter-regional migration in Europe. Environment and Planning A, 45:1491-1507.

Durrett, R. and Levin, S. 1994. The importance of being discrete (and spatial). Theoretical Population Biology, 46:363-394.

Epstein, J. M. 1997. Nonlinear dynamics, mathematical biology, and social science. Addison-Wesley, Reading, MA. 
Fry, H. and Wilson, A. G. 2012. A dynamic global trade model with four sectors: food, natural resources, manufactured goods and labour. CASA Working Paper, 178.

González, E. and Villena, M. 2011. Spatial Lanchester models. European Journal of Operational Research, 210(3):706-715.

Harris, B. and Wilson, A. 1978. Equilibrium values and dynamics of attractiveness terms in productionconstrained spatial-interaction models. Environment and Planning A, 10:371-388.

Ilachinski, A. 2004. Artificial War: Multiagent-Based Simulation of Combat. World Scientific Publishing Co. Pte. Ltd., Singapore.

Intriligator, M. D. and Brito, D. L. 1988. A predator-prey model of guerrilla warfare. Synthese, 2:235-244.

Jackson, S., Russett, B., Snidal, D., and Sylvan, D. 1978. Conflict and coercion in dependent states. Journal of Conflict Resolution, 22(4):627-657.

Karmeshu, M., Jain, V., and Mahajan, A. 1990. A dynamic model of domestic political conflict process. Journal of Conflict Resolution, 34(2):252-269.

Keane, T. 2011. Combat modelling with partial differential equations. Applied Mathematical Modelling, 35 (6):2723-2735.

Kress, M. and MacKay, N. J. 2014. Bits or shots in combat? The generalized Deitchman model of guerrilla warfare. Operations Research Letters, 42:102-108.

Lanchester, F. W. 1916. Aircraft in Warfare: The Dawn of the Fourth Arm. Constable and Company Limited, London.

Liebovitch, L. S., Naudot, V., Vallacher, R., Nowak, A., Bui-Wrzosinska, L., and Coleman, P. 2008. Dynamics of two-actor cooperation-competition conflict models. Physica A: Statistical Mechanics and its Applications, 387(25):6360-6378.

Lotka, A. J. 1925. Elements of Physical Biology. Williams \& Wilkins Company, Baltimore. 
Mandelbrot, B. 1967. How long is the coast of britain? Statistical self-similarity and fractional dimension. Science, 156(3775):636-638.

Pitcher, A. B. 2010. Adding police to a mathematical model of burglary. European Journal of Applied Mathematics, 21:401-419.

Protopopescu, V. and Santoro, R. 1989. Combat modeling with partial differential equations. European Journal of Operational Research, 38:178-183.

Qubbaj, M. and Muneepeerakul, R. 2012. Two-actor conflict with time delay: A dynamical model. Physical Review E, 86(5):056101.

Richardson, L. F. 1952. Contiguity and deadly quarrels: The local pacifying influence. Journal of the Royal Statistical Society. Series A (General), 115(2):219-231.

Richardson, L. F. 1960. Arms and Insecurity. The Boxwood Press, Pittsburgh, PA.

Richardson, L. F. 1961. The problem of contiguity; an appendix to statistics of deadly quarrels. General Systems Yearbook, 6:140-87.

Rojas-Pacheco, A., Obregón-Quintana, B., Liebovitch, L. S., and Guzmán-Vargas, L. 2013. Time-delay effects on dynamics of a two-actor conflict model. Physica A: Statistical Mechanics and its Applications, 392(3):458-467.

Saperstein, A. M. 2007. Chaos in Models of Arms Races and the Initiation of War. Complexity, 12(3).

Senior, M. 1979. From gravity modelling to entropy maximising: a pedagogic guide. Progress in Human Geography, 3:175-210.

Short, M. B., Bertozzi, A., and Brantingham, P. 2010a. Nonlinear patterns in urban crime: Hotspots, bifurcations and suppression. SIAM Journal of Applied Dynamical Systems, 9(2):462-483. 
Short, M. B., Brantingham, P. J., Bertozzi, A. L., and Tita, G. E. 2010b. Dissipation and displacement of hotspots in reaction-diffusion models of crime. Proceedings of the National Academy of Sciences, 107(9): $3961-3965$.

Smith, L. M., Keegan, M. S., Wittman, T., Mohler, G. O., and Bertozzi, A. L. 2010. Improving density estimation by incorporating spatial information. EURASIP Journal on Advances in Signal Processing, 2010:265631.

Tobler, W. R. 1970. A computer model simulating urban growth in the Detroit region. Economic Geography, 46:234-240.

Wilson, A. G. 1967. A statistical theory of spatial distribution models. Transportation Research, 1.

Wilson, A. G. 1970. Entropy in Urban and Regional Modelling. Pion, London, UK.

Wilson, A. G. 2006. Ecological and urban systems models: Some explorations of similarities in the context of complexity theory. Environment and Planning A, 38:633-646.

Wilson, A. G. 2008. Boltzmann, Lotka and Volterra and spatial structural evolution: An integrated methodology for some dynamical systems. Journal of the Royal Society, Interface / the Royal Society, 5(25): 865-71.

Zinnes, D. A. and Muncaster, R. G. 1984. The dynamics of hostile activity and the prediction of war. Journal of Conflict Resolution, 28(2):187-229. 